\title{
GPR Image Decomposition Using Two Dimensional Singular Spectrum Analysis
}

\author{
Branislav Vuksanovic \\ School of Engineering \\ University of Portsmouth \\ Portsmouth, UK \\ branislav.vuksanovic@port.ac.uk
}

\begin{abstract}
Ground penetrating radar measurements can suffer from large amount of noise and clutter. Current methods, such as time gating and background averaging, mostly applied to remove reflections from air-ground interface do not perform well when removal of extraneous and very strong and non-uniform clutter signals originating from the objects in the surveyed area other than the target is needed. This work describes and evaluates performance of Singular Spectrum Analysis (SSA) and its multivariate derivatives for those tasks. Experimental GPR data using simple geometric shapes measured under laboratory conditions are used to demonstrate the effectiveness of proposed algorithm for these tasks.
\end{abstract}

Keywords-ground penetrating radar; clutter; target; two dimensional singular spectrum analysis;

\section{INTRODUCTION}

The term Ground Penetrating Radar (GPR) refers to a range of electromagnetic (EM) techniques designed primarily for the location of objects or interfaces beneath the earth's surface or within a visually opaque structure [1]. GPR is a nondestructive and non-invasive geophysical technique with a wide variety of applications in site and ground investigations. It is mostly used in reflection mode where a signal is emitted via a transmitter antenna into the structure and soil under investigation. Energy reflected by changes in material properties is received and recorded by the receiver antenna as shown in Fig 1. The collected raw data are then processed and interpreted [2].

The signal reflected of the target can be weak and overlapped with clutter, defined as group of signals that are uncorrelated to the target scattering characteristics but occupy the same frequency band as the target [3, 4], making it difficult to distinguish between both (signals and clutter) without applying suitable signal processing techniques.

The interference in measured GPR signals is usually made up of three main components [5]:

(i) The cross-talk between the transmitting and receiving antennas,

(ii) The reflection from the air-ground interface,

(iii) The scattered signals from other objects within the soil.

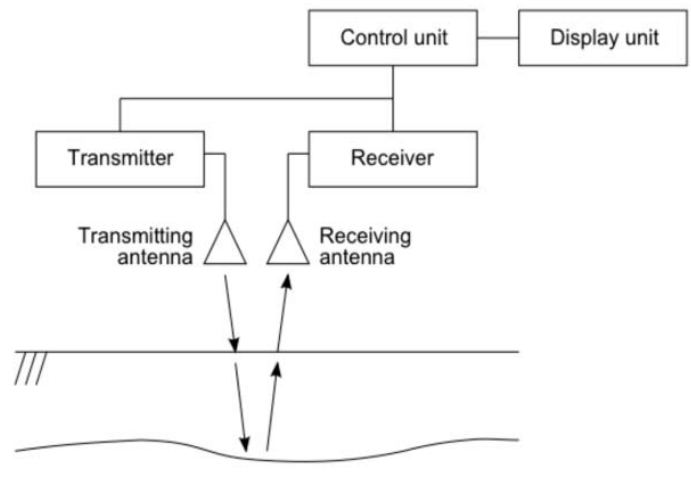

Fig. 1. Elements of Ground Penetrating Radar System

However, due to the constant distance between both antennas, the cross-talk signal (i) will arrive to antenna receiver earlier than any other signal which makes it easier to distinguish and reject this "interference" component [6]. The air-ground interface portion of the signal (ii) is usually very strong and a number of clutter reduction approaches have been suggested in the literature, including simple mean scan subtraction [7], two-dimensional digital filtering [8], wavelet packet decomposition [7], likelihood ratio test [6, 7, 9], parametric system identification [7, 10], and Kalman filtering $[9,11]$. Most of these methods depend on background signal estimation [6] where the background signal is estimated by taking the mean value of the unprocessed ensemble of the collected GPR data, followed by employing the simple mean scan subtraction. A-scan, a trace of returned radar signal far away from the target location might be used instead of mean scan subtraction. These methods have been used widely in GPR applications, but the work on this problem is ongoing as they cannot completely remove the direct wave from the received data due to the air-earth interface and time-shift (jitter) of the radar system.

Removing signals (iii) scattered from other objects within the soil is a difficult problem and cannot be effectively tackled with previously listed methods. Those features are usually unevenly distributed across the image and can in many cases be much stronger and overlap - spatially and spectrally with the weak target signals. To extract the target signals from such 
a complex data set, this work proposes the use of a relatively new data decomposition technique - Singular Spectral Analysis (SSA). SSA is closely related to more established Principal Component Analysis method and relies on reducing the higher-order statistical dependencies in GPR returned signals. Using the modified versions of SSA - multivariate and two dimensional singular spectral analysis (M-SSA and 2D-SSA) techniques, GPR images can effectively be decomposed into clutter and useful signals by selecting a set of extracted components. Selected components can then be used to determine the subspace for reconstruction of a GPR data set containing significantly reduced clutter components.

Various researchers have shown interest in subspace techniques including the Singular Value Decomposition (SVD) [7, 12], Linear Discriminant Analysis (LDA) [13], PCA [14, 15], and ICA [4, 14, 16, 17]. The PCA uses a second-order statistics to decompose measured data set into two orthogonal matrices that convey useful information about the main components contained in the data set. PCA also provides a third, diagonal matrix where diagonal elements of this matrix indicate the amount of information (variance) contained in each principal component. This makes it relatively easy to decide which components to discard in the reconstruction process of the GPR image, based on the values of the diagonal elements in this matrix. On the other side, components extracted via PCA technique are orthogonal and therefore not easily physically interpreted, which can represent a problem in processing results of physical measurements such are GPR data sets. The ICA technique minimises the statistical dependence between the data and uses higher-order statistics to provide more meaningful information about data. Components returned by ICA are however scaled in amplitude and usually randomly permutated so the task of identifying the "most important" components in the extracted set is not a trivial one.

The Singular Spectrum Analysis (SSA) [18], [19] is a model-free technique for the analysis of time series, closely related to previously discussed multivariate techniques. In the recent years, it has been successfully applied to a different areas of science, engineering and economy. The name of this method implies the use of singular value decomposition algorithm; where set of obtained eigenvalues and corresponding eigenvectors is added together to either obtain suitable decomposition of time series or to extract component with particular oscillatory behaviour from time series. Some of the tasks SSA is especially well suited in solving include finding trends of different resolution, smoothing, extraction of seasonality components, simultaneous extraction of cycles with small and large periods, extraction of periodicities with varying amplitudes, finding structure in short time series and change-point detection. Three versions of the SSA algorithm will be briefly explained in the following section of this paper; the basic, univariate SSA algorithm, multivariate SSA method (M-SSA) and a slightly modified version of M-SSA algorithm suitable for image analysis and decomposition, known as two- dimensional SSA (2D-SSA) will be reviewed.

The rest of the paper is organized in the following way. Section 2 provides a brief overview of three SSA algorithms. Section 3 describes the measurement setup used to collect the experimental GPR data containing strong non-uniform clutter. Section 4 presents results achieved with the proposed algorithm and the final section summarises the completed work and draws some conclusions and recommendations for further research into the problems of target characterisation for GPR signals.

\section{SingUlAR SPECTRUM ANALYSIS ALgORITHMS}

\section{A. One Dimensional SSA}

SSA algorithm consists of two complementary stages decomposition and reconstruction of the given data set with each of those two stages again performed through two separate steps. Decomposition is implemented via data embedding stage followed by the singular value decomposition of the embedded data. Reconstruction comprises grouping and diagonal averaging stages. Details of those sub-stages are described below.

\section{1) Embedding}

Basic SSA algorithm treats the univariate, i.e. onedimensional time series of length $L, Y_{L}=\left(y_{1}, y_{2}, \ldots, y_{L}\right)$. Embedding stage transforms this series into multi-dimensional series, matrix of the form $\mathbf{X}=\left[X_{1}, X_{2}, \ldots, X_{K}\right]$ where columns of this matrix, i.e. vectors $X_{i}, i=1, \ldots, K$ are so called lagged vectors, $X_{i}=\left(y_{i}, y_{i+1}, \ldots, y_{i+M-1}\right)^{T}$. Length of the lagged vectors is determined by the choice of embedding parameter $M$, which is usually called window length, since the process of embedding can be viewed as sliding the window of length $M$ along the time series, extracting lagged vectors and arranging them into matrix $\mathbf{X}$. Matrix $\mathbf{X}$ obtained through this process is Henkel type matrix and in the context of SSA is usually referred to as trajectory matrix. Using an embedding window of size $M$, will produce an $M \times K$ size trajectory matrix, where $K=L-M+1$ is parameter determined by the choice of window length $M$.

2) SVD

Second step of decomposition part of SSA algorithm performs SVD on generated trajectory matrix $\mathbf{X}$ to obtain three new matrices $\mathbf{U}, \mathbf{V}$ and $\boldsymbol{\Sigma}$ :

$$
\mathbf{X}=\mathbf{U} \boldsymbol{\Sigma} \mathbf{V}^{T}
$$

Alternatively, matrices $\mathbf{U}, \mathbf{V}$ and $\boldsymbol{\Lambda}$ can be obtained via eigendecomposition of matrix products $\mathbf{X} \mathbf{X}^{T}$ and $\mathbf{X}^{T} \mathbf{X}$ since :

$$
\mathbf{X} \mathbf{X}^{T}=\mathbf{U} \boldsymbol{\Sigma} \mathbf{V}^{T}\left(\mathbf{U} \boldsymbol{\Sigma} \mathbf{V}^{T}\right)^{T}=\mathbf{U} \boldsymbol{\Sigma} \mathbf{V}^{T} \mathbf{V} \boldsymbol{\Sigma} \mathbf{U}^{T}=\mathbf{U} \boldsymbol{\Sigma}^{2} \mathbf{U}^{T}(2)
$$
and 


$$
\mathbf{X}^{T} \mathbf{X}=\left(\mathbf{U} \boldsymbol{\Sigma} \mathbf{V}^{T}\right)^{T} \mathbf{U} \boldsymbol{\Sigma} \mathbf{V}^{T}=\mathbf{V} \boldsymbol{\Sigma} \mathbf{U}^{T} \mathbf{U} \boldsymbol{\Sigma} \mathbf{V}^{T}=\mathbf{V} \boldsymbol{\Sigma}^{2} \mathbf{V}^{T}
$$

Elements of matrix $\boldsymbol{\Sigma}$ are non-negative values, known as the singular values of $\mathbf{X}$, while the columns of matrices $\mathbf{U}$ and rows of matrix $\mathbf{V}$ represent the eigenvectors of matrices $\mathbf{X} \mathbf{X}^{T}$ and $\mathbf{X}^{T} \mathbf{X}$ respectively. The diagonal matrix $\boldsymbol{\Sigma}^{2}$, usually denoted as $\boldsymbol{\Lambda}$ now contains the corresponding eigenvalues $\lambda_{i}$ for the eigenvectors in $\mathbf{U}$ and $\mathbf{V}$, i.e.

$$
\boldsymbol{\Sigma}^{2}=\operatorname{diag}\left\{\lambda_{1}, \lambda_{2}, \ldots, \lambda_{n}\right\}=\operatorname{diag}\left\{\sigma_{1}^{2}, \sigma_{2}^{2}, \ldots, \sigma_{n}^{2}\right\}
$$

Individual matrices $\mathbf{E}_{i}$, corresponding to each $\mathbf{u}_{i}, \mathbf{v}_{i}$ set of eigenvectors from $\mathbf{U}$ and $\mathbf{V}$ can be obtained using:

$$
\mathbf{E}_{i}=\mathbf{u}_{i} \mathbf{v}_{i}^{T}
$$

and the SVD of trajectory matrix $\mathbf{X}$ represented as the summation of those matrices:

$$
\mathbf{X}=\sum_{i=1}^{M} \sigma_{i} \mathbf{E}_{i}
$$

\section{3) Grouping}

Grouping stage is the first stage of the reconstruction part of SSA and involves grouping of the elementary matrices $\mathbf{E}_{i}$ into $P$ groups and summing of the matrices from each group. This stage basically starts by splitting the set of indices $i=1,2, \ldots, M$ into a number of disjointed sets. Selection and number of those is usually done manually, by the user but can be difficult and cumbersome for the large data sets. This paper proposes grouping technique suitable for GPR image analysis and decomposition. Elementary matrices corresponding to indices from each set are then added together to form a new group of matrices $\mathbf{D}_{j}, j=1,2, \ldots P$.

\section{4) Diagonal Averaging}

Diagonal averaging is the process of turning each grouped matrix $\mathbf{D}_{j}$ obtained in the previous, grouping stage back into time series. Obtained time series are the additive components of the initial time series $Y_{L}$. The $k$-th element of the time series corresponding to matrix $\mathbf{D}$ can be obtained by averaging elements $d_{i, j}$ of this matrix over all indices $i$ and $j$ such that $i+j=k+2$. This procedure is known as diagonal averaging or Hankelisation of a matrix $\mathbf{D}$. Once each $\mathbf{D}_{j}$ matrix is turned into time series, those can be added back to recover the original time series $Y_{L}$.

More formally, process of diagonal averaging can be described using equation:

$$
y_{k}=\left\{\begin{array}{cc}
\frac{1}{k} \sum_{m=1}^{k} d_{m, k-m+1}^{*} & \text { for } 1 \leq k<L^{*} \\
\frac{1}{L^{*}} \sum_{m=1}^{L^{*}} d_{m, k-m+1}^{*} & \text { for } L^{*} \leq k \leq K^{*} \\
\frac{1}{N-k+1} \sum_{m=k-K^{*}+1}^{N-K^{*}+1} d_{m, k-m+1}^{*} & \text { for } K^{*}<k \leq N
\end{array}\right.
$$

where $\quad K=N-L+1, \quad L^{*}=\min (L, K) \quad$ and $K^{*}=\max (L, K)$.

\section{B. Multichannel SSA (M-SSA)}

M-SSA is an extension of SSA algorithm which can be applied to multivariate time series, i.e. when more than one time series is available for analysis. Algorithm can be demonstrated on the simple example where two time series or the recordings from two channels are to be analysed together. If we denote two data sets as $Y_{L}=\left(y_{1}, y_{2}, \ldots, y_{L}\right)$ and $X_{L}=\left(x_{1}, x_{2}, \ldots, x_{L}\right)$, than each of those can be embedded to create its own trajectory matrix. Individual trajectory matrices $\mathbf{T}_{Y}$ and $\mathbf{T}_{X}$ corresponding to each channel are therefore created as in the SSA algorithm. The total trajectory matrix can then be formed as:

$$
\mathbf{T}=\left[\begin{array}{ll}
\mathbf{T}_{Y} & \mathbf{T}_{Y}
\end{array}\right]^{T}
$$

and further steps, identical to the ones in SSA method performed. This idea can then be easily extended to any number of channels. If, in more general case $Q$ channels $Y_{j}, j=1,2, \ldots, Q$, each of length $L$ are available, and stored in matrix $\mathbf{Y}$ with dimensions $L \times Q$, trajectory $\mathbf{T}_{j}$ matrix for each channel $Y_{j}$ from this matrix can be formed and combined into a total trajectory matrix $\mathbf{T}$ :

$$
\mathbf{T}=\left[\begin{array}{llll}
\mathbf{T}_{1} & \mathbf{T}_{2} & \ldots & \mathbf{T}_{Q}
\end{array}\right]^{T}
$$

It is worth noting that trajectory matrices formed in the first stage of M-SSA procedure are not Hankel format matrices any more but instead have a so called block-Hankel structure. After generation of a total trajectory matrix, M-SSA procedure follows the rest of the univariate SSA steps - SVD decomposition of trajectory matrix, grouping and diagonal averaging.

\section{Two-dimensional SSA (2D-SSA)}

Two dimensional singular spectrum analysis [20] is similar to M-SSA algorithm but whilst the M-SSA algorithm usually considers one dimension of data matrix $\mathbf{Y}$ to be time, 2D-SSA is designed to process more general two-dimensional scalar fields. In practice, this involves using a two-dimensional instead of one-dimensional data window, moving it along all available positions in data matrix $\mathbf{Y}$ and generating a trajectory matrix for each position of this window in the matrix. 
Individual trajectory matrices are then combined into total trajectory matrix and the standard SSA steps followed.

If for the embedding purposes we adopt the window of size $M_{H} \times M_{W}$, where $M_{H}$ and $M_{W}$ represent window height and width respectively, than placing the top left corner of this window in the position of element $y_{i, j}$ in data matrix $\mathbf{Y}$ where:

$$
\mathbf{Y}=\left[\begin{array}{cccc}
y_{1,1} & y_{1,2} & \cdots & y_{1, Q} \\
y_{2,1} & y_{2,2} & & y_{2, Q} \\
\vdots & & \ddots & \vdots \\
y_{L, 1} & y_{L, 2} & \cdots & y_{L, Q}
\end{array}\right]
$$

results in the extraction of submatrix $\mathbf{Y}_{i, j}$ such that:

$$
\mathbf{Y}_{i, j}=\left[\begin{array}{cccc}
y_{i, j} & y_{i, j+1} & \ldots & y_{i, i+M_{W}-1} \\
y_{i+1, j} & y_{i+1, j+1} & & y_{i+1, i+M_{W}-1} \\
\vdots & & \ddots & \vdots \\
y_{i+M_{H}-1, j} & y_{i+M_{H}-1, j+1} & \ldots & y_{i+M_{H}-1 i+M_{W}-1}
\end{array}\right]
$$

Just as in the case of basic, one-dimensional SSA algorithm, where embedding stage produces trajectory matrix, i.e. given $1 \mathrm{D}$ time series gets transformed into $2 \mathrm{D}$ data structure (matrix), embedding data matrix $\mathbf{Y}$ into equivalent trajectory structure would in general case require moving from 2D data structure to 4D. To avoid this complexity, and keep working with 2D matrices, submatrix $\mathbf{Y}_{i, j}$ is flattened, i.e. reshaped into column and used as an $\left((i-1) K_{W}+j\right)$ th column of the total trajectory matrix, providing we move the top left corner of our data window left to right and top to bottom through the matrix $\mathbf{Y}$. Constant $K_{W}=\left(L-M_{W}\right)+1$ indicates the number of available movements from the left to right of data matrix. Similarly constant $K_{H}=\left(L-M_{H}\right)+1$ indicates the number of valid window positions when the window moves from top to the bottom of data matrix. Using this notation, it is easy to see that the complete trajectory matrix $\mathbf{T}$ created from data matrix $\mathbf{Y}$ will have $K_{H} \cdot K_{W}$ columns, with $M_{H} \cdot M_{W}$ elements in each column arranged in the following way:

$$
\mathbf{T}=\left[\begin{array}{lllllllll}
\mathbf{y}_{1,1} & \mathbf{y}_{1,2} & \ldots & \mathbf{y}_{1, K_{C}} & \mathbf{y}_{2,1} & \ldots & \mathbf{y}_{2, K_{C}} & \ldots & \mathbf{y}_{K_{H}, K_{C}}
\end{array}\right]
$$

2D-SSA algorithm further follows the classical SSA steps SVD, grouping of obtained eigenmatrices and Hankelisation of each group to obtain corresponding submatrix of the original data matrix $\mathbf{Y}$. Due to a different structure of trajectory matrix $\mathbf{T}$, diagonal averaging procedure is slightly modified for the case of 2D-SSA algorithm. Dimensions of data matrix $\mathbf{Y}$ treated in this work are relatively high, thus a large number of eigenmatrices is usually produced after SVD. To prevent cumbersome manual grouping of those matrices automatic grouping technique is proposed and described further in the paper.

It is also worth noting that the 2D-SSA algorithm represents more general algorithm in comparison to M-SSA, SSA and SVD algorithms. If the 2D embedding window becomes one-dimensional, i.e. $M_{W}=1$, 2D-SSA in effect becomes M-SSA. On the other hand, if both widow dimensions become $1, M_{H}=M_{W}=12 \mathrm{D}$-SSA reduces to SVD. For onedimensional set of data, i.e. when $Q=1$ and $M_{W}=1$, 2DSSA reduces to classic SSA operation.

\section{GPR MEASUREMENTS AND EXPERIMENTAL DATA COLLECTION}

Aim of conducted experiment was to evaluate the capabilities of $4 \mathrm{GHz}$ GPR for detecting and characterising samples of three PVC pipes of different diameters $(11 \mathrm{~cm}, 4.5$ $\mathrm{cm}$. and $3 \mathrm{~cm}$ ). To perform the experiment, $120 \times 120 \times 15 \mathrm{~cm}$ open-top wooden box was constructed and 4 different sets of measurements carried out. GPR measurements included (i) empty box (i.e. no pipes and no other material in the box), (ii) box containing each of pipe samples individually and all three pipes together but no other material in the box, (iii) empty box filled with sand but no pipes in the box and (iv) the box with pipes buried in the sand. To scan the content of the constructed wooden box, $2 \mathrm{~cm}$ thick wooden panel was placed on the top of the box and scans along the $10 \mathrm{~cm}$ grid drawn on the top panel performed. $4 \mathrm{GHz}$ Grandvue 5 GPR device manufactured by Utsi Electronics Ltd has been used to perform all measurements in this work. Fig 2. shows the setup and GPR equipment used in the experiment.

Obtained results after some basic pre-processing are illustrated using the GPR image corresponding to, "left to right" scan along line 7 of the measurement grid (i.e. scan directly across the centres of all or each pipe sample in the box). To reduce the amount of clutter corresponding to airwood panel interface and antenna cross talk effects strongly present in both raw images, mean trace was calculated and removed from all images used in this study before any further processing is attempted. No further processing or image enhancement has been performed on measured data at this stage. GPR images with removed mean trace made on empty box, the box containing all three pipes and single pipe in the box without sand are shown in Fig 3.

Whilst the emergence of two hyperbolas corresponding to big $(11 \mathrm{~cm})$ and medium $(4.5 \mathrm{~cm})$ diameter pipes can be observed in Fig 3b) the main feature present in both images is the clutter - strong signal on the left side of both images indicated with black arrows. This is caused by the reflection of the EM waves from the left, side of the wooden box. Similar feature, corresponding to the reflection from the right side of the box (white arrows) can also be observed on the right side of the image, although it is slightly weaker due to larger distance between the end of the scanned line and right side of the wooden box. 

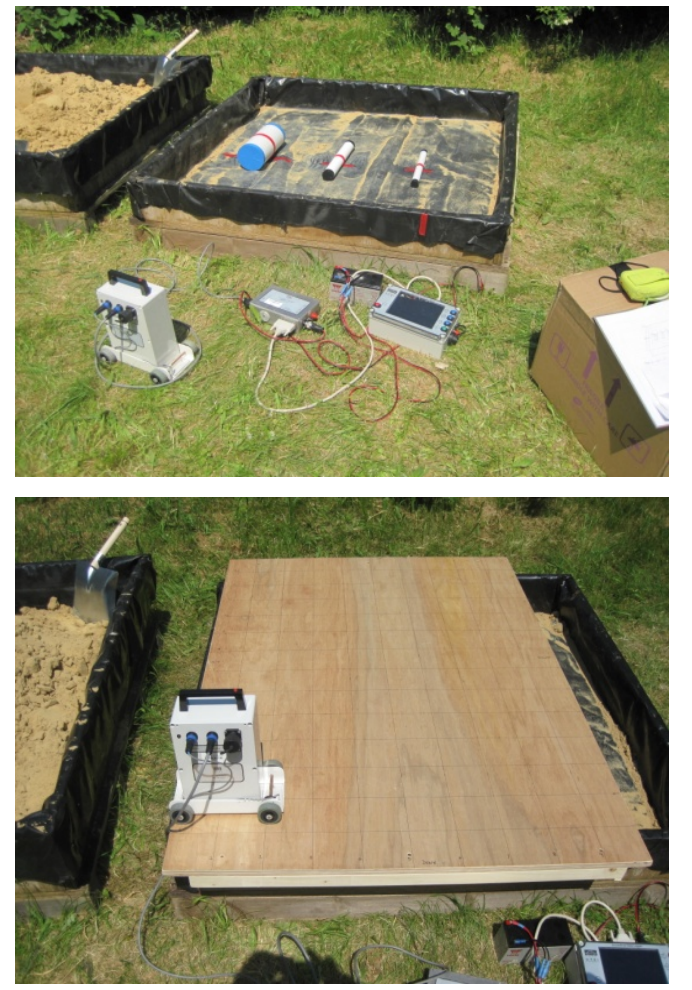

Fig. 2. Experimental setup and GPR equipment used in the experiment

a)
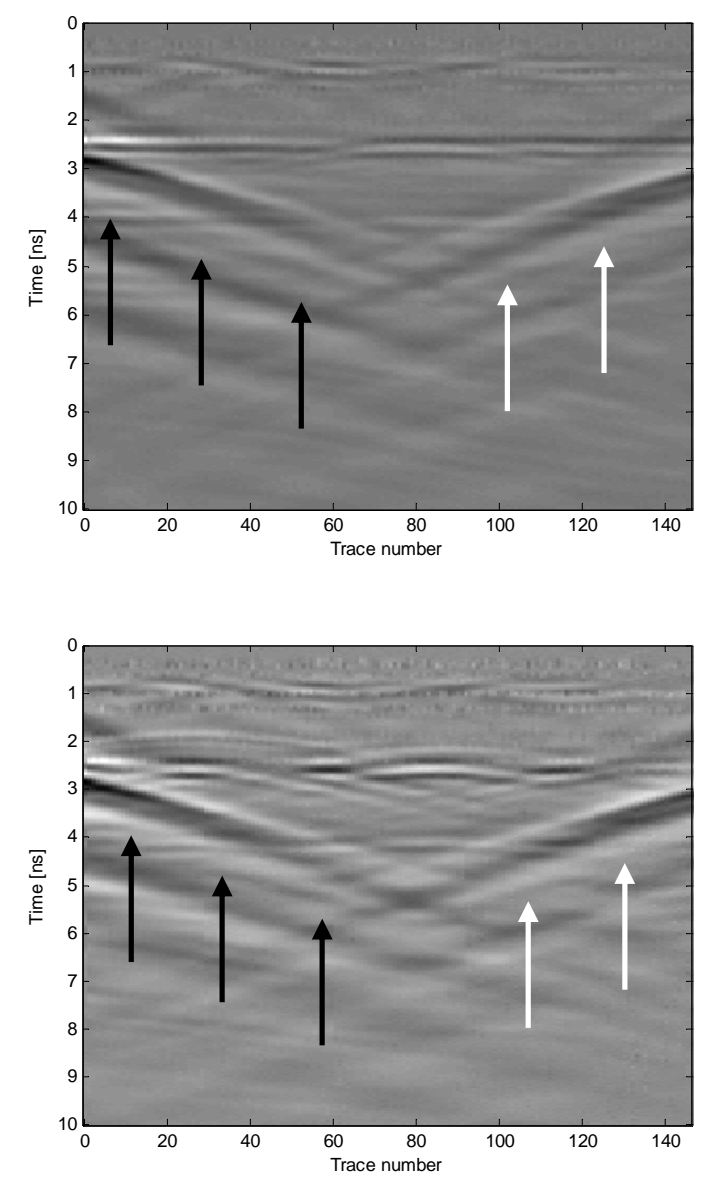

b)

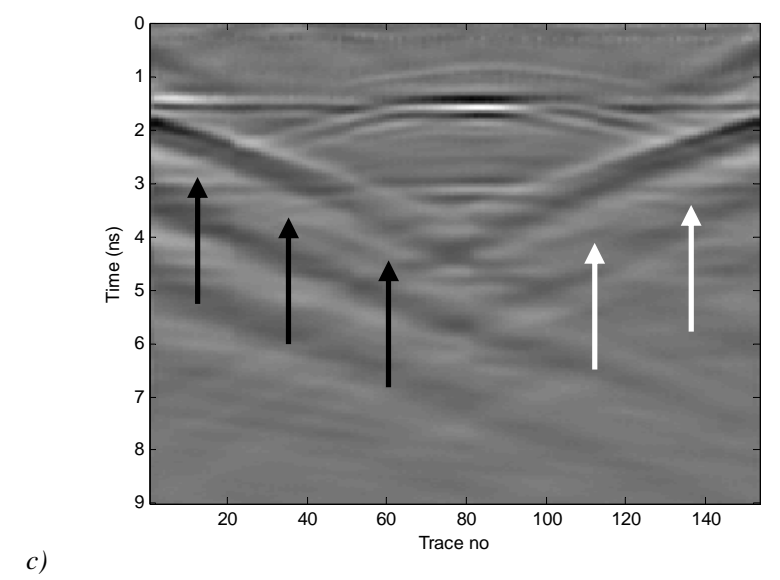

Fig. 3. GPR images with removed mean trace measured on a) empty box, b) box containing three plastic pipes and c) single pipe in the box

It is however strong enough to completely obscure most of the hyperbola feature emanating from the third and smallest, right most positioned pipe in the box. Both side reflections are evidently the strongest features in the image and as such they heavily obscure the hyperbolas corresponding to plastic pipes to be detected and characterised. It would therefore be beneficial to be able to "blindly" identify those features in the image with minimal effects on the content of the rest of the image, before attempting to detect and analyse target (i.e. pipe) related features in the image. Unlike reflections from the airground or in this case air- wood panel interface, reflections from the side of the box are non-uniform and as such present a good example of strong clutter, reflection from non-target objects in the scanned area. Absence of other material filling the box (e.g. sand) makes the presence of unwanted side reflections even stronger thus those images were selected to test the proposed SSA based techniques and its capabilities in separating and eventually removing this type of clutter from the measured GPR data. Results are illustrated and discussed in the following section. Analysis of decluttered images, i.e. pipe detection and characterisation will be tackled in the extension of this work and described in the subsequent publication.

\section{RESUlts}

Due to a space limitation only the results of image decomposition using 2D-SSA algorithm are presented in this section. Following the 2D-SSA procedure outlined in the previous section GPR image measured on the box containing a single pipe shown in Fig 3c). has been decomposed and 30 subimages, corresponding to 30 highest eigenvalues from matrix $\boldsymbol{\Lambda}$ retained for grouping stage. Manual inspection and grouping of those images is a difficult and time-consuming task, thus the extracted subimages were classified into four groups using the eigenfaces-like, PCA based feature extraction and dimensionality reduction algorithm [21] followed by kmeans clustering of a reduced dimensionality data set. Results, 4 images obtained by adding all images from each cluster are shown in Fig 4. Axis labels in this figure have been left out as they are identical to the ones shown in Fig 3. It is worth noting that the number of classes specified in k-means clustering procedure call is not a critical parameter in this process. 
Following the automatic grouping of SSA subimages, further merging of some of those images can be easily done manually and even smaller number of subimages, representing the clutter from various, multiple sources in the surveyed area obtained.

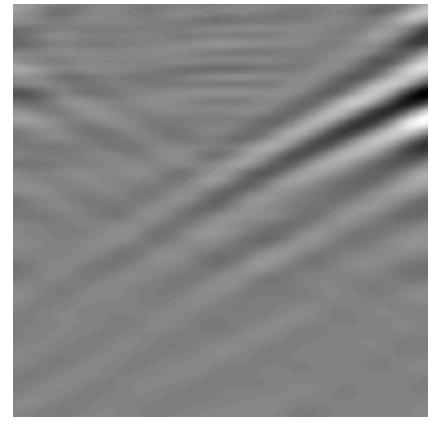

a)

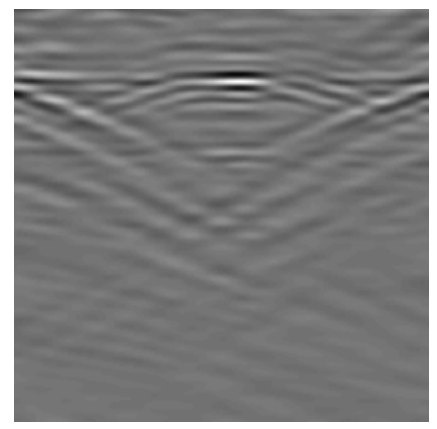

c)

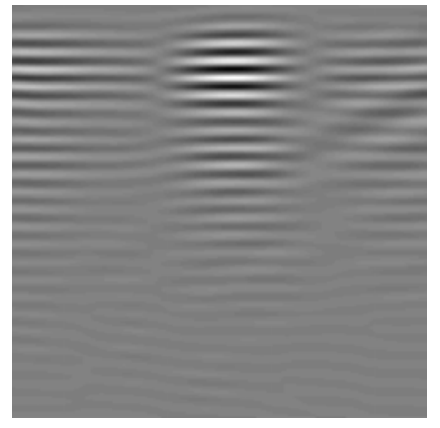

b)

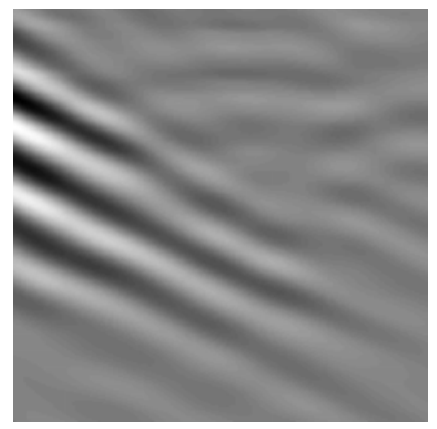

d)
Fig. 4. Sub-images extracted using 2D-SSA and grouped following clustering of all obtained images

Sub-images shown in Fig 4. indicate potential of 2D-SSA algorithm to separate components of the original image. Fig 4a) clearly contains clutter mainly corresponding to reflections of the radar waves from the right side of the container box, whilst the Fig 4d) contains slightly stronger clutter corresponding to reflections from the left side of the box. Figs $4 \mathrm{~b})$ and $4 \mathrm{c}$ ) therefore correspond to target/pipe reflections. Those two images can be added together and analysed to extract target properties and characterise targets. Alternatively, each of those two images could be analysed individually with the same aim.

\section{ACKNOWLEDGMENT}

This work was supported by Sustainable Society Network grant.

\section{REFERENCES}

[1] Zhao, A., Y. Jiang, and W. Wang, Exploring Independent Component Analysis for GPR Signal Processing. PIERS Online, 2005. 1(6): p. 750753.

[2] Alani, A., Preliminary Investigation of Detection of Leakage from Joints and Cracks in Water Reservoirs Using Ground Penetrating Radar, 2007.
[3] Daniels, D.J., Surface-penetrating radar. Electronics \& Communication Engineering Journal, 1996. 8(4): p. 165-182.

[4] Zhao, A., Y. Jiang, and W. Wang. Exploring Independent Component Analysis for GPR Signal Processing. in Progress In Electromagnetics Research Symposium 2005, Hangzhou, China. 2005.

[5] Brooks, J.W., The Detection of Buried Non-Metallic Anti-Personnel Land Mines, in Department of Electrical and Computer Engineering2000, University of Alabama: Huntsville. p. 127.

[6] Nadim, G., Clutter reduction and detection of landmine objects in ground penetrating radar data using likelihood method. 2008 3rd International Symposium on Communications, Control and Signal Processing, Vols 1-3, 2008: p. 98-106.

[7] Abujarad, F., A. Jostingmeier, and A.S. Omar, Clutter removal for landmine using different signal processing techniques. Proceedings of the Tenth International Conference on Ground Penetrating Radar, Vols 1 and 2, 2004: p. 697-700.

[8] Potin, D., E. Duflos, and P. Vanheeghe, Landmines ground-penetrating radar signal enhancement by digital filtering. Ieee Transactions on Geoscience and Remote Sensing, 2006. 44(9): p. 2393-2406.

[9] van Kempen, L. and H. Sahli, Signal Processing Techniques for Clutter Parameters Estimation and Clutter Removal in GPR Data for Landmine Detection. 2001 Ieee Workshop on Statistical Signal Processing Proceedings, 2001: p. 158-161.

[10] van der Merwe, A. and I.J. Gupta, A novel signal processing technique for clutter reduction in CPR measurements of small, shallow land mines. Ieee Transactions on Geoscience and Remote Sensing, 2000. 38(6): p. 2627-2637.

[11] Zoubir, A.M., et al., Signal processing techniques for landmine detection using impulse ground penetrating radar. Sensors Journal, IEEE, 2002. 2(1): p. 41-51.

[12] Abujarad, F., G. Nadim, and A. Omar, Clutter reduction and detection of landmine objects in ground penetrating radar data using Singular Value Decomposition (SVD). Proceedings of the 3rd International Workshop on Advanced Ground Penetrating Radar, 2005: p. 37-41.

[13] Belhumeur, P.N., J.P. Hespanha, and D.J. Kriegman, Eigenfaces vs. Fisherfaces:Recognition using class specific linear projection. Pattern Analysis and Machine Intelligence, IEEE Transactions on, 1997. 19(7): p. 711-720.

[14] Abujarad, F. and A. Omar, GPR data processing using the componentseparation methods PCA and ICA. Ist 2006: Proceedings of the 2006 IEEE International Workshop on Imaging Systems and Techniques, 2006: p. 59-63.

[15] Karlsen, B., et al., Comparison of PCA and ICA based clutter reduction in GPR systems for anti-personal landmine detection. 2001 IEEE Workshop on Statistical Signal Processing Proceedings, 2001: p. 146149.

[16] Karlsen, B., et al., Independent component analysis for clutter reduction in Ground Penetrating Radar data. Detection and Remediation Technologies for Mines and Minelike Targets Vii, Pts 1 and 2, 2002. 4742: p. 378-389.

[17] Gao, Q., T. Li, and R.B. Wu, A novel KICA method for ground bounce removal with GPR. Proceedings of 2006 CIE International Conference on Radar, Vols 1 and 2, 2006: p. 1058-1061.

[18] Golyandina, N. and A. Zhigljavsky, Singular Spectrum Analysis for Time Series. SpringerBriefs in Statistics. 2013: Springer.

[19] Hassani, H., Singular Spectrum Analysis: Methodology and Comparison. Journal of Data Science, 2007. 5: p. 239-257.

[20] Rodriguez-Aragon, L.J. and A. Zhigljavsky, Singular spectrum analysis for image processing. Statistics and Its Interface, 2010. 3: p. 419-426.

[21] Belhumeur, P.N., J.P. Hespanha, and D.J. Kriegman, Eigenfaces vs. Fisherfaces: recognition using class specific linear projection. Pattern Analysis and Machine Intelligence, IEEE Transactions on, 1997. 19(7): p. 711-720. 